\title{
Correction to: Rethinking Image Inpainting via a Mutual Encoder-Decoder with Feature Equalizations
}

Hongyu Liu, Bin Jiang, Yibing Song, Wei Huang, and Chao Yang

\begin{abstract}
Correction to:
Chapter "Rethinking Image Inpainting via a Mutual

Encoder-Decoder with Feature Equalizations"

in: A. Vedaldi et al. (Eds.): Computer Vision - ECCV 2020,

LNCS 12347, https://doi.org/10.1007/978-3-030-58536-5_43
\end{abstract}

In the originally published version of chapter 43 , the second affiliation stated a wrong city and country. This has been corrected. 